\title{
Emerging Non-invasive Raman Methods in Process Control and Forensic Applications
}

\author{
Neil A. Macleod ${ }^{1}$ and Pavel Matousek ${ }^{1,2}$
}

Received February 5, 2008; accepted April 1, 2008; published online April 16, 2008

\begin{abstract}
This article reviews emerging Raman techniques (Spatially Offset and Transmission Raman Spectroscopy) for non-invasive, sub-surface probing in process control and forensic applications. New capabilities offered by these methods are discussed and several application examples are given including the non-invasive detection of counterfeit drugs through blister packs and opaque plastic bottles and the rapid quantitative analysis of the bulk content of pharmaceutical tablets and capsules without sub-sampling.
\end{abstract}

KEY WORDS: forensic; non-invasive; pharmaceutical; process control; Raman.

\section{INTRODUCTION}

In a number of analytical applications in the pharmaceutical industry it is a requirement that the chemical constituency of a product is analysed rapidly, non-destructively and non-invasively with minimal sample preparation or pretreatment. Applications span the entire process from production (process control) through to quality control and counterfeit detection of packaged products.

Raman spectroscopy holds particular promise in this area due to its inherent high chemical specificity (substantially higher than that of near-infrared absorption (NIR) spectroscopy), the ability to probe samples in the presence of water and its potential for high penetration depth into nonabsorbing or weakly absorbing turbid samples such as pharmaceutical powders (similar to that of NIR absorption spectroscopy). The higher chemical specificity of Raman spectroscopy relative to NIR absorption stems from the fact that NIR spectra consist of broad (overlapping) overtone and combination bands of fundamental vibrational modes. In contrast, Raman spectra exhibit much sharper bands corresponding to fundamental vibrational modes (1). Although the optical counterparts of Raman scattering (mid-infrared (MIR) and terahertz (THz) spectroscopy) also offer high chemical specificity, their applications are typically restricted to dry samples due to strong water absorption. In addition, MIR spectroscopy is generally only capable of probing very thin layers of samples unless the samples are substantially diluted. On the other hand, the Raman technique suffers from high sensitivity to fluorescence which can swamp the

\footnotetext{
${ }^{1}$ Central Laser Facility, Science and Technology Facilities Council, Rutherford Appleton Laboratory, Harwell Science and Innovation Campus, Didcot, Oxfordshire, OX11 0QX, UK.

${ }^{2}$ To whom correspondence should be addressed. (e-mail: P.Matousek@ rl.ac.uk)
}

much weaker Raman signal. This problem can, however, often be circumvented by using near-infrared excitation to avoid excitation into fluorescing states (1).

The Raman effect is the inelastic scattering of photons from molecules via interactions with the vibrational modes within the molecule. In this process, the photon energy and consequently the photon wavelength are altered (1) giving a spectral signature unique to each chemical species undergoing scattering. Most commonly the photon loses energy and its wavelength is shifted, typically by several hundreds to thousands of wavenumbers away from the laser wavelength, towards longer wavelengths. The Raman shift, or energy loss, is directly related to the frequencies of vibrational motion activated within the molecule which are themselves highly dependent on chemical constitution; thus the identity and structure of the molecule can be deduced.

Historically, the Raman method has been used with commercial Raman probes and microscopes predominantly in the backscattering collection mode (see Fig. 1) although in a number of specialist laboratories a $90^{\circ}$ collection geometry can also be found (1). The backscattering geometry is widely used for its instrumental simplicity and ease of deployment. Its major drawback is an inability to probe deep layers of turbid samples such as pharmaceutical tablets or capsules (1) where the effective probed depths are typically only a millimetre or two (2).

Recently, a substantial increase of the Raman signal penetration depth was accomplished by utilising the diffuse component of light rather than the ballistic component (3). In conjunction with temporal and spatial gating methods (and in analogy with NIR absorption tomography (4-7) or fluorescence spectroscopy (8-11)) this allows Raman spectra of sublayers to be identified. These developments are beginning to shed new light into the potential use of Raman spectroscopy throughout the pharmaceutical industry from production and quality control through to shelf-life determination and counterfeit detection. 
These newly emerging developments, in common with other more established spectroscopic techniques, require statistical analysis to extract the required information (chemical composition, concentration of analyte, etc.) from the extensive, spectrally complex, datasets produced. While a detailed review of the application of such multivariate techniques to process monitoring and the pharmaceutical industry in general is beyond the scope of this article, a brief outline is useful. In spectroscopic terms, multivariate analysis assumes a linear dependence between spectral response and concentration which, in favourable cases, allows decomposition of a dataset ( $m$ spectra recorded in $n$ wavelength channels) into two simpler matrices corresponding to concentration $(k$ by $m)$ and pure spectra $(k$ by $n)$ where $k$ is the number of distinct analytes in the dataset. Additional information from external sources (e.g. concentration from "wet" chemical analysis) can be added to build calibration models (e.g. using partial least squares (PLS) or principal component regression (PCR)) which are subsequently applied to unknown samples for quantitative prediction. For a more detailed overview of multivariate methods see (12).

\section{METHODS FOR DEEP RAMAN SPECTROSCOPY OF TURBID MEDIA}

\section{Temporal Gating}

A major obstacle preventing conventional Raman spectroscopy from acquiring signals from deep areas of turbid samples is the presence of intense Raman signals originating from the surface layers of the probed sample which mask the weaker subsurface signals. The Raman signals from deeper zones are typically substantially diluted due to the wide diffusion of laser and Raman photons away from the vicinity of the collection/deposition area due to the long pathways required to sample the target layer. The overwhelming surface signals can be effectively discriminated against using impulsive Raman excitation and fast (picosecond) temporal gating of the backscattered Raman signal as demonstrated by Wu et al. (13) who used a photon counting approach to accomplish gated detection and imaging of a single Raman component. The recovery of the full Raman spectrum of a deeply buried layer in a turbid sample was demonstrated using the Kerr gating approach by Matousek et al. (14) on a two-layer powder sample; this work combined pioneering research into Raman photon migration (Everall et al. $(15,16)$ and Morris et al. (17)) with fluorescence rejection techniques (Matousek et al. (18-20)). A more extensive description of these methods and their underlying mechanisms can be found in (3). Although these methods were demonstrated to be technically viable the instrumental complexity and high peak intensities of ultrafast lasers precluded their wider practical usage.

\section{Spatially Offset Raman Spectroscopy}

The instrumental complexity and laser intensity issues were addressed through the development of a spatial equivalent to temporal gating known as Spatially Offset Raman Spectroscopy (SORS) $(21,22)$. With this concept the Raman spectra of individual sub-layers within a complex multi-layer system can be isolated with a considerably smaller and simpler experimental approach which replaces the ultrafast laser with a continuous, low power diode laser and removes the need for fast temporal gating or photon counting (see Fig. 1).

The SORS concept is straightforward; Raman signal is collected from areas that are spatially offset (by a distance $\Delta s$ ) from the point of illumination (see Fig. 2a). The key point is that spectra collected at each spatial offset contain different relative contributions from sample layers located at different depths due to the wider spread of photons originating from deeper layers on the sample surface. The lateral offset also effectively discriminates against photons propagating sideways within the surface layers as they exhibit a higher loss at the air-to-sample interface than photons propagating through deeper layers. Consequently, the SORS technique effectively suppresses the interfering Raman (and fluorescence) signals originating from the surface layers, e.g. capsule shell, enhancing, in relative terms, the sub-layer Raman signal, e.g. a pharmaceutical product contained within the capsule shell.

The availability of a set of Raman spectra with varying degrees of surface and subsurface contributions permits a complete isolation of the Raman spectrum belonging to individual layers. This can be accomplished using a variety of numerical multivariate methods including band target entropy minimization (BTEM) $(23,24)$. For a "simple" two layer system, this can also be achieved by a manual or automated scaled subtraction of two SORS spectra obtained at different spatial offsets to cancel the surface contribution $(25,26)$. This is in contrast with conventional backscattering Raman spectroscopy where the solitary Raman spectrum available is typically dominated by intense surface layer components and no information is available to permit the separation of the surface and subsurface Raman components even if subsurface Raman signals are detectable. Elimination of the surface layer with conventional Raman spectroscopy can only be performed if the Raman spectrum of the pure over-layers is known a priori and if the surface layer Raman signal does not swamp the underlying subsurface Raman component, conditions which are rarely satisfied simultaneously in deep subsurface Raman spectroscopy.

The SORS method was first demonstrated on a stratified powder sample (21) and has been used since in numerous practical applications including the non-invasive Raman spectroscopy of bones (25), in pharmaceutical forensic applications (27) and security screening (26). The first demonstration was carried out on a two-layer sample com-

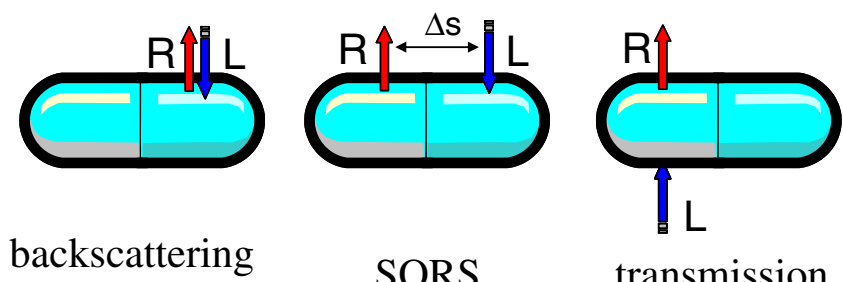

Fig. 1. Basic types of Raman spectroscopy geometries with respect to the sample: Conventional backscattering Raman, SORS and Raman transmission geometries. $R$ Raman light, $L$ laser beam. 

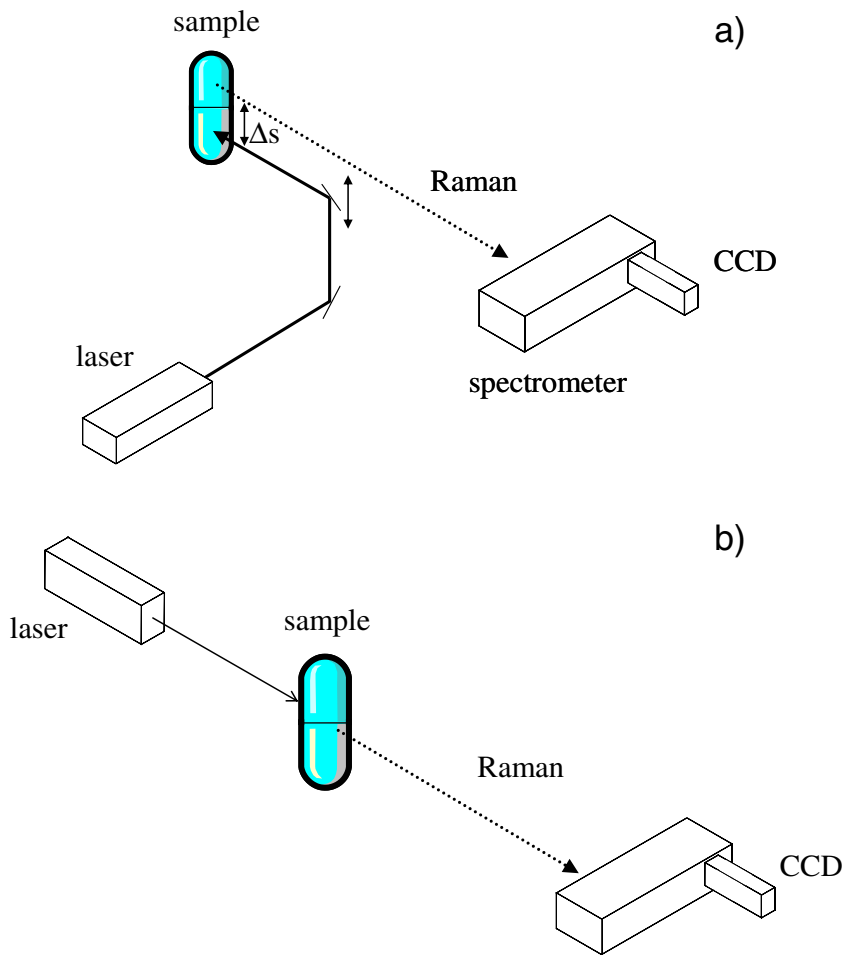

spectrometer

Fig. 2. Schematic diagram of a basic implementation of a the SORS and $\mathbf{b}$ transmission Raman geometries.

posed of a $1 \mathrm{~mm}$ thick PMMA powder layer placed on top of a layer of trans-stilbene powder (21). The results of these measurements are shown in Fig. 3. The conventional Raman spectrum is represented by the zero-offset. The introduction of the spatial offset leads to a much faster decay of the surface layer Raman signal (PMMA) compared with the sub-layer (trans-stilbene). The article reports an improvement in subsurface-to-surface relative Raman intensity by a factor of 19 at a spatial offset of $3.5 \mathrm{~mm}$ (21).

In order to maximise the signal quality the SORS concept is often combined with a fibre optic collection scheme developed for conventional Raman spectroscopy by Ma and Ben-Amotz (28). In this scheme fibres collecting Raman signal from the sample are arranged in a ring or disk pattern and are re-organised on the spectrograph slit into a linear pattern matching the spectrograph slit geometry (21,22,29-31). Similar concepts were also used in fluorescence tomography (8-11).

A simple variant of SORS implementable on conventional Raman systems was recently described by Eliasson et al. (32) and relies on defocusing a conventional Raman collection system to induce a change in the relative Raman contributions from surface and subsurface layers. Although less effective than conventional SORS it can be used with less challenging samples and provides a convenient first stepping stone for researchers in possession of conventional Raman instruments wishing to initiate research in this area.

More recently a refined SORS method, inverse SORS, was developed permitting the use of higher overall laser powers through the provision of much wider illumination zones on the sample. This concept is particularly beneficial when probing living tissue in vivo or pharmaceutical samples in explosive powder environments where stringent illumination intensity limits exist.

The inverse SORS concept was demonstrated independently by Matousek (33) and Schulmerich et al. (34,35). In contrast to conventional SORS, this approach is based on the collection of Raman light through a group of fibres contained within the centre of a probe and the delivery of the laser beam through a ring shaped beam area. The ring radius of the beam defines the SORS spatial offset. The concept also permits the setting of an arbitrary illumination radius enabling better tailoring of the experimental conditions to sample parameters than with conventional SORS probes where collection ring radii are built into the fibre probe and their radius cannot be altered by simple means. The ringshaped laser beam is typically generated using a conical lens (axicon) (36) and the spatial offset is set by adjusting the distance of the axicon from the sample (33) or by varying the magnification of a telescope inserted between the sample and the axicon $(34,35)$.

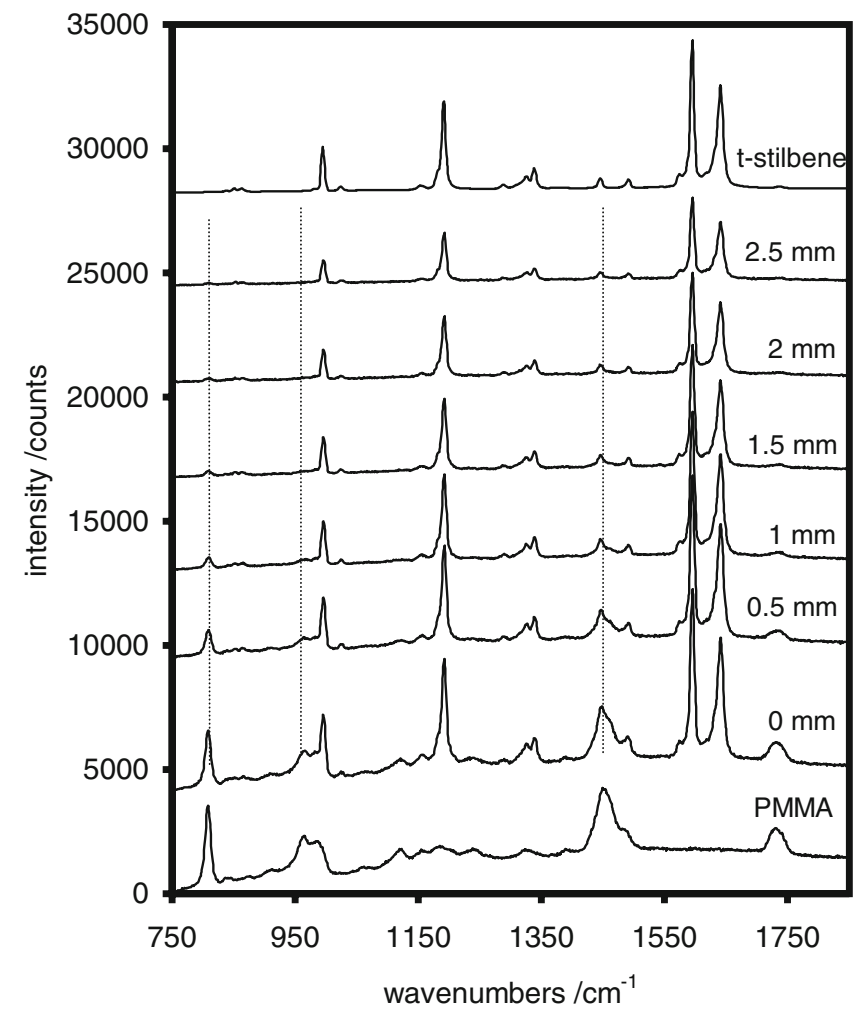

Fig. 3. A set of SORS spectra collected from a two-layer system consisting of $1 \mathrm{~mm}$-thick layer of PMMA made of $20 \mu \mathrm{m}$-diameter spheres followed by a $2 \mathrm{~mm}$-layer of trans-stilbene powder measured using $514 \mathrm{~nm}$ as the probe wavelength. The spectra are shown for different spatial offsets. The top and bottom spectra are those of the individual layers obtained in separate measurements. The spectra are offset for clarity. The acquisition time was $100 \mathrm{~s}$ for each spectrum and the average laser power $12 \mathrm{~mW}$. (Reprinted with permission from P. Matousek, I. P. Clark, E. R. C. Draper, M. D. Morris, A. E. Goodship, N. Everall, M. Towrie, W. F. Finney and A. W. Parker, Appl. Spectrosc. 59, 393-400 (2005). Copyright (2005) The Society for Applied Spectroscopy (21)). 


\section{Transmission Raman}

Another deep Raman spectroscopy concept, related to SORS, uses a transmission geometry to provide information on the bulk content of samples through a single Raman exposure (2). In this method, the laser beam impacts on one side of the sample and the Raman signal is collected from the opposite side (see Fig. 1). Although the transmission Raman technique was demonstrated in the very early days of Raman spectroscopy (37), its benefits for the non-invasive probing of the bulk content of pharmaceutical products have not been recognised. In particular, these include the removal of the sub-sampling problem (38) (the inability to probe deeper layers of turbid samples) and the effective suppression of interfering fluorescence and Raman signals originating from surface layers (39). This concept can be considered to be a special case of SORS with the illumination and collection points displaced to the maximum extent.

Sub-sampling is a particular obstacle for conventional backscattering Raman spectroscopy of pharmaceutical formulations which are often highly heterogeneous (38). To reduce this effect, special devices for rotating pharmaceutical tablets in front of a conventional backscattering Raman spectrometer are used. More recently a large illumination probe was developed by Kaiser Optical Systems (PhAT) to minimise this issue by collecting Raman signal from a large surface area of the sample although the penetration depth of this concept is still insufficient to provide bulk information on a typical tablet or capsule (2).

The transmission Raman concept (see Fig. 2b) eliminates the need for any special rotators as it inherently provides the Raman signal originating from the entire bulk of the probed object. With this concept, bulk information on a tablet or capsule can be obtained rapidly within a single spectral acquisition permitting a rapid deployment of this method in process analytical applications on production lines.

The elimination of the sub-sampling problem in this geometry was confirmed by Monte Carlo simulations by Matousek and Parker (2). The results of this study are shown in Fig. 4 and illustrate that if a thin 'impurity' layer is re-

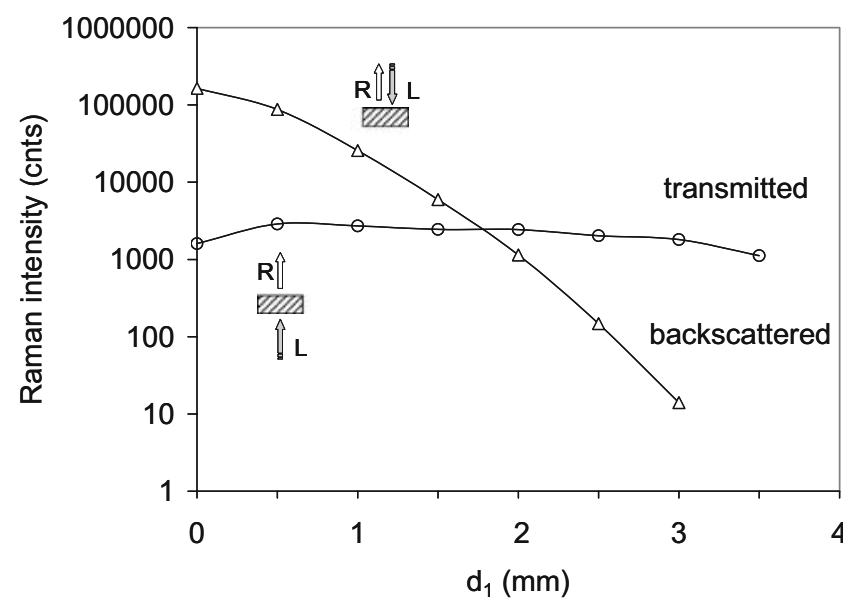

Fig. 4. Plot of Raman intensities for the backscattering and transmission geometries versus depth $\left(d_{1}\right)$ of the inter-layer (impurity) within a pharmaceutical tablet-like medium. The dependencies are the results of Monte Carlo simulations. (Reprinted with permission from P. Matousek, A.W. Parker, Appl. Spectrosc. 2006, 60, 1353-1357. Copyright (2006) The Society for Applied Spectroscopy (2)). located from the surface to a depth of $3 \mathrm{~mm}$ within a typical tablet medium its conventional backscattering Raman signal is diminished by 4 orders of magnitude. In practical terms, such signal is lost in the dominant Raman signal originating from the surface layers. On the other hand, in the transmission Raman geometry the signal is largely insensitive to the depth from which it originates.

The experimental demonstration of the elimination of the sub-sampling problem was carried out by Matousek and Parker (2) on a standard paracetamol tablet of $3.9 \mathrm{~mm}$ thickness with a simulated impurity layer (2 mm trans-stilbene powder layer) placed either in front or behind the tablet relative to the illuminating laser beam. The measurements were performed using an $830 \mathrm{~nm}$ probe, with a laser beam diameter of $4 \mathrm{~mm}$. The results are shown in Fig. 5 and demonstrate a strong sub-sampling problem in the conventional geometry which yields predominately Raman signatures of the surface layers probed by the instrument. In contrast, the transmission geometry provided Raman signals originating from both the tablet and the 'impurity' and their relative intensity was largely independent of the position of the impurity relative to the tablet; a result in good agreement with the theoretical simulations.

Due to the passage of photons through the entire thickness of tablet the overall Raman signal in the transmission mode is smaller than in the backscattering geometry. Intuitively, one might assume that it would be negligible given the tablet thickness. However, the observed signal diminishment for a typical tablet of $3.9 \mathrm{~mm}$ thickness was observed to be only a factor of 12 . The fact that a relatively high level of Raman signal remains after propagating through such a thick turbid medium is due to the fact that the Raman component diminishes more slowly than its elastic (laser) counterpart; while both components exhibit decay due to photon diffusion, the Raman component is partially regenerated from the laser component (Everall et al. (15)).

Enhancement of the transmission Raman signal, if desired, can be accomplished by increasing either the laser power (which is permitted by the relatively large illumination areas possible with the transmission geometry) and/or using 'unidirectional' coupling mirrors as described below (40). These improvements have the potential to reduce acquisition times to fractions of a second in many practical situations making transmission Raman spectroscopy an attractive candidate for many PAT applications.

In many applications, it is also beneficial that the transmission Raman method inherently suppresses fluorescence emission originating from the surface layers of the probed sample, in contrast, with the conventional backscattering Raman geometry which accentuates such interfering signals (39).

\section{Raman Signal Enhancement Using a 'Unidirectional' Mirror}

When probing pharmaceutical tablets, Raman signals in SORS and transmission Raman spectroscopy can be substantially enhanced using a 'unidirectional' mirror (Matousek (40)) leading to higher spectral quality, enhanced penetration depth and shorter acquisition times. The special mirror consists of a multilayer dielectric element placed over the 

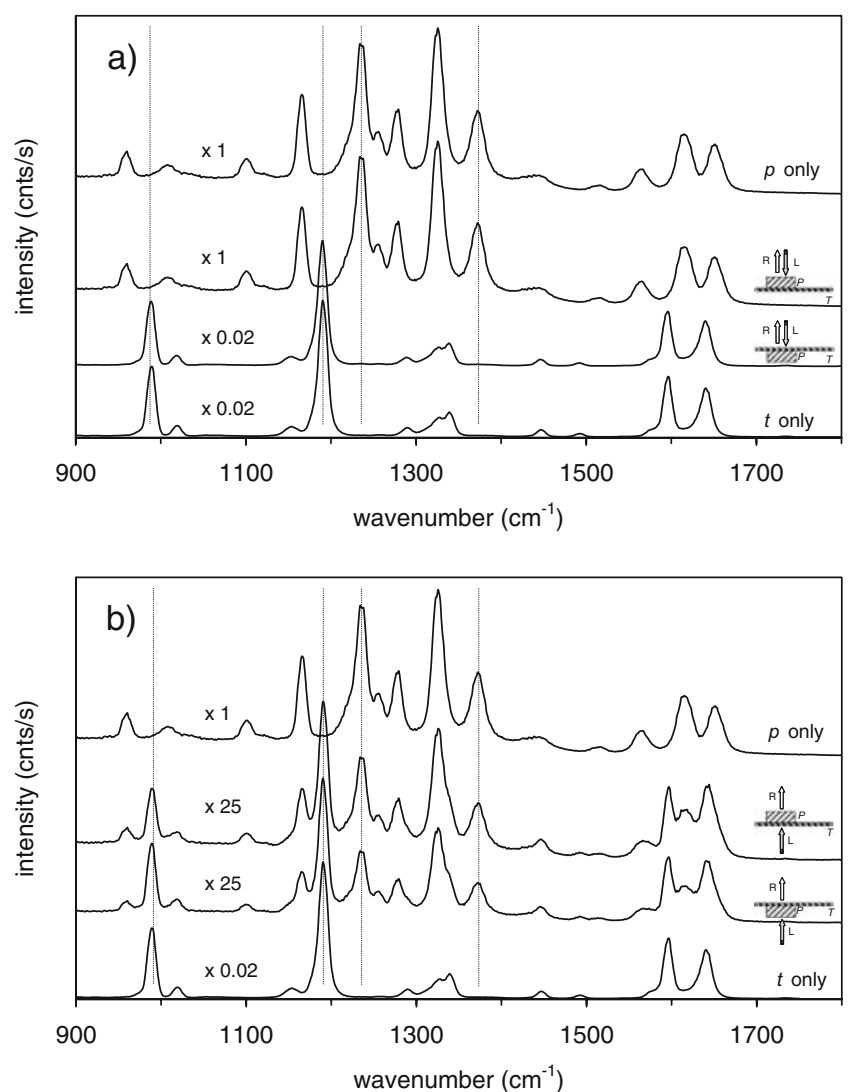

Fig. 5. The Raman spectra obtained from a two-layer sample (3.9 $\mathrm{mm}$ thick paracetamol tablet and $2 \mathrm{~mm}$ thick trans-stilbene powder 'impurity' layer) using a conventional backscattering geometry and $\mathbf{b}$ transmission geometry. The measurements are performed at two sample orientations, with paracetamol at the top and bottom of the trans-stilbene cell as indicated in the graphs. The top and bottom spectra are those of paracetamol and trans-stilbene, respectively, obtained in separate experiments. The acquisition times were between 0.2 and $10 \mathrm{~s}$, with a laser power of $80 \mathrm{~mW}$. The spectra are offset for clarity. $P$ paracetamol, $T$ trans-stilbene, $R$ Raman light, $L$ laser beam. (Reprinted with permission from P. Matousek and A.W. Parker, Appl. Spectrosc. 2006, 60, 1353-1357. Copyright (2006) The Society for Applied Spectroscopy (2)).

laser illumination zone in close proximity to the sample. The element is in essence a narrow bandpass filter designed to transmit semi-collimated laser radiation at normal incidence. The concept is based on the angular dependence of the dielectric filter spectral properties on impacting photon direction. This spectral profile shifts to lower wavelength with increasing deviation of photons away from normal incidence and enables the element to act as a unidirectional mirror passing a semi-collimated laser beam through unhindered at one side whilst, at the other side, reflecting photons emerging from the sample at random directions (mostly away from normal incidence). This prevention of photon loss, which is often the dominant photon loss mechanism, leads to a substantial increase of the overall Raman signal. In its basic form, the method requires that the coupling sample surface is not heavily curved. Consequently the method, without taking any further measures, is readily applicable to flat pharmaceutical tablets while much lower effectiveness is observed for curved objects such as capsules.
Figure 6 shows the results of a feasibility experiment carried out in transmission Raman geometry on a standard paracetamol tablet with and without a bandpass filter (transmission centered at $830 \mathrm{~nm}$ with bandwidth (FWHM) of $3.2 \mathrm{~nm}$ ). An eight-fold enhancement was observed upon the insertion of the bandpass filter into the proximity of a two-layer powder sample. The enhancement was observed to be present for both layers with the surface layer experiencing slightly larger enhancement, by about $13 \%$.

Such large enhancements could be difficult, and in many cases impossible, to reproduce by other means. It is also worth noting that the signal enhancement is uniform across the entire spectrum and no spectral fluctuations are present. The presence of enhancement was also experimentally verified with the SORS concept where Raman signal detected
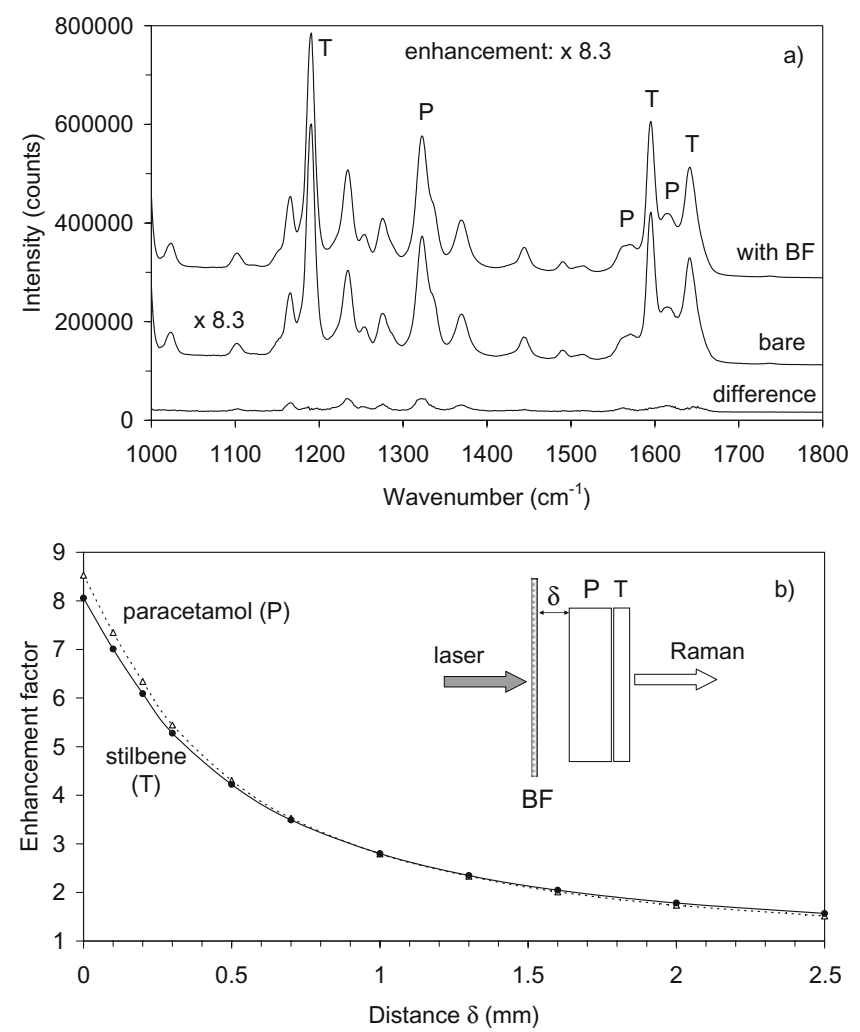

Fig. 6. The experimental demonstration of the Raman signal enhancement concept on a two-layer system. The Raman spectra were obtained from a two-layer sample composed of a $3.8 \mathrm{~mm}$ thick paracetamol tablet $(P)$ and $2 \mathrm{~mm}$ thick trans-stilbene powder layer $(T)$ in transmission geometry. The trans-stilbene layer was facing the Raman collection system and the bandpass filter BF was in contact with the paracetamol tablet. a Raman spectra measured with (with $B F$ ) and without (bare) the bandpass filter BF. A difference spectrum (difference) obtained by scaled subtraction of the Raman spectra with and without the enhancing filter cancelling the trans-stilbene contribution is also shown. The residual spectrum is that of paracetamol indicating that this layer has experienced somewhat higher enhancement. b The dependence of Raman signal enhancement factor of the two individual layers on the distance of the bandpass filter from the sample. The acquisition times in all the measurements were $0.5 \mathrm{~s}$ with a laser power of $250 \mathrm{~mW}$ $(830 \mathrm{~nm})$. The spectra are offset for clarity. (Reprinted with permission from P. Matousek, Appl. Spectrosc. 2007, 61, 845-854. Copyright (2007) The Society for Applied Spectroscopy (40)). 
at the detection limit was enhanced and its signal to noise substantially improved (41).

\section{EXAMPLES OF PHARMACEUTICAL APPLICATIONS}

\section{Forensic Applications}

One major area where SORS has proved to be particularly useful is the authentication of pharmaceutical products through non-invasive chemical analysis of the content of opaque plastic bottles and other types of packaging. The method offers a unique potential to inspect and chemically identify products throughout the entire supply chain in a non-invasive and non-destructive manner. The need for such inspection has heightened in recent years with the increase of counterfeit products on the market (42). The targeted drugs in the developed world often include lifestyle drugs and drugs for chronic diseases. More seriously, in Africa and Asia, it is the essential life-saving medicines, such as anti-infective drugs, which are plagued by this growing problem (43-45).

Raman techniques offer an attractive alternative to current methods (principally NIR spectroscopy) in which subtle spectral differences in composition are often hard to detect and chemometric tools are routinely required for data interpretation. In addition, NIR cannot be deployed noninvasively in many cases because of the interference and absorption of NIR signal by common packaging materials.

As an alternative technique, Raman spectroscopy provides substantially higher chemical specificity and better penetration depth. The method in its conventional form permits the interrogation of many pharmaceutical products through their coatings or capsules as well as through blister packs and has also been used to detect counterfeit drugs (46). However, in many instances, and in particular with darkly colored coatings or capsules, or opaque packaging, the Raman signal of the API can be heavily polluted with fluorescence and Raman signals originating from the coating, capsule (39) or blister pack itself. The interfering signals reduce the sensitivity of the technique and can preclude the observation of the API and other ingredients altogether.

Recently Eliasson and Matousek $(27,47)$ demonstrated that SORS can provide a chemical signature of the internal content of opaque plastic containers. This is demonstrated in Fig. 7 for aspirin tablets held inside an opaque (white) plastic pharmaceutical bottle (1.3 $\mathrm{mm}$ thick). The conventional Raman signal is overwhelmed by the Raman component originating from the container wall and is consequently ineffective in determining the contents of the bottle. In contrast, the SORS method using a scaled subtraction of two SORS spectra measured at different spatial offsets yields a clean Raman spectrum of the tablets inside the bottle. The experiments were performed at $830 \mathrm{~nm}$ with a $250 \mathrm{~mW}$ laser beam and an acquisition time of $1 \mathrm{~s}$.

More recently, SORS was also used in the detection of counterfeit anti-malarial tablets by Ricci et al. (48). As the SORS concept can be readily incorporated into existing commercial hand-held Raman instruments it holds great promise for more accurate and sensitive interrogation of drugs throughout the entire supply chain.

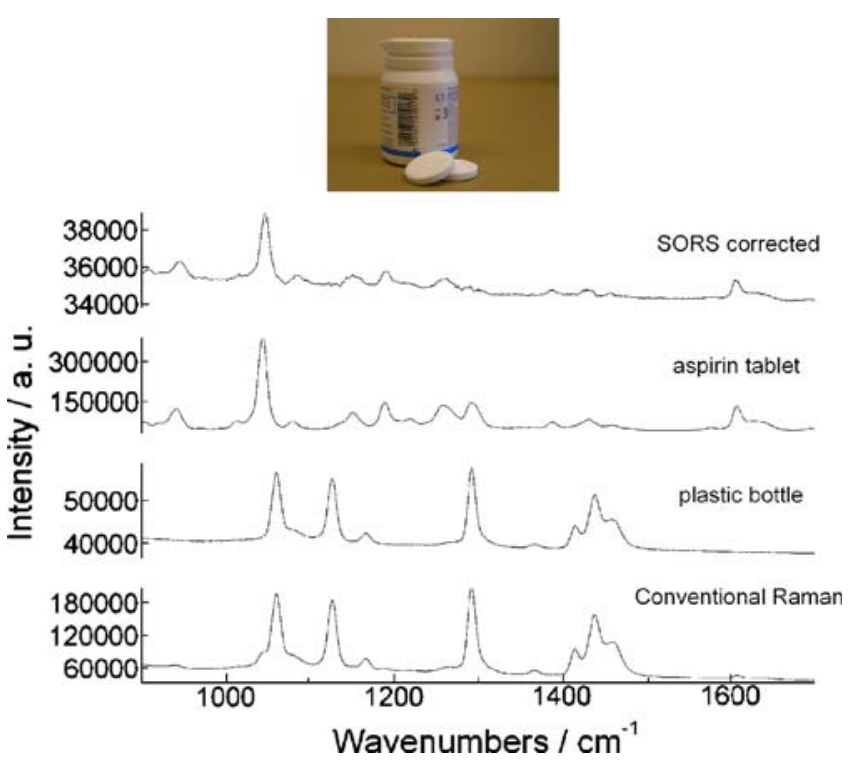

Fig. 7. Non-invasive Raman spectra of aspirin tablets measured through a white, diffusely scattering $1.3 \mathrm{~mm}$ thick plastic container. Conventional Raman and the scaled and subtracted SORS data are shown together with the reference Raman spectra of aspirin and the plastic container. The conventional Raman spectrum is dominated by the Raman signal of the container masking the Raman signal of the aspirin contents. The acquisition time was $1 \mathrm{~s}$ and the laser beam power $250 \mathrm{~mW}$.

\section{Probing of Pharmaceutical Tablets and Capsules in Process Control}

A number of pharmaceutical process analytical technology (PAT) applications require the monitoring of the bulk content of pharmaceutical products. Ideally, this should be accomplished quickly, non-destructively and non-invasively with high chemical specificity. Although NIR absorption spectroscopy is widely used in this area, in a number of applications it has insufficient chemical specificity and is hampered by the presence of water. In addition, tablet coatings and capsule shells, in particular those containing $\mathrm{TiO}_{2}$, can present an insurmountable challenge. As discussed earlier the transmission Raman approach effectively overcomes such hurdles (2) making it a formidable contender for displacing NIR absorption spectroscopy in number of application areas.

A study by Matousek and Parker (39) demonstrates that the transmission Raman geometry also dramatically reduces fluorescence interference originating from surface layers of the probed sample, thus permitting more sensitive spectroscopic interrogation of the internal capsule chemical content. The results of comparative measurements using transmission and conventional Raman geometries on a variety of coloured capsules are shown in Fig. 8. The spectra are displayed in their raw form with no background subtraction. The data demonstrates that interfering surface fluorescence, for many coloured capsules, significantly deteriorates the overall quality of conventional Raman spectra. On the other hand, the transmission Raman method effectively reduces this interference permitting much more sensitive measurement of the internal content. 

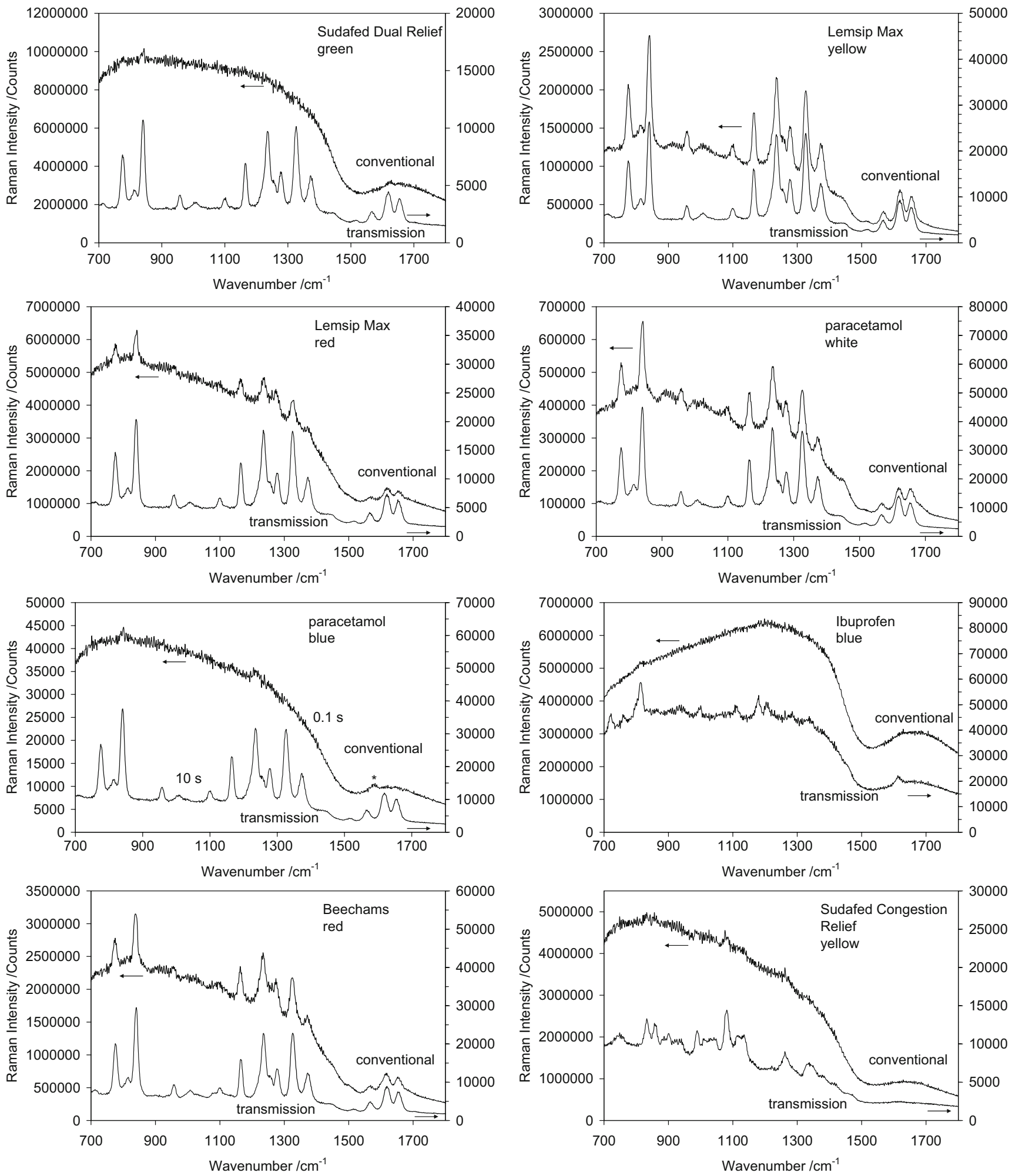

Fig. 8. The comparison of performance of the conventional backscattering and transmission Raman geometries in probing various pharmaceutical capsules. The acquisition times were $10 \mathrm{~s}$ unless indicated otherwise, with a laser power of $80 \mathrm{~mW}$. Asterisk designates Raman band originating from capsule shell. (Reprinted with permission from P. Matousek, A.W. Parker, Non-Invasive Probing of Pharmaceutical Capsules using Transmission Raman Spectroscopy, J. Raman Spectrosc. 2007, 38, 563-567. Copyright (2007). (C) John Wiley \& Sons (39)).

The ability of the transmission Raman approach to determine the quantitative chemical composition of pharmaceutical tablets and capsules was recently demonstrated by Johansson et al. (49) using 20 test tablets of thickness $3.3 \mathrm{~mm}$ prepared under laboratory conditions. In this study the active pharmaceutical ingredient (API) was quantified with a relative root mean square error of prediction of $+1-2.2 \%$ in tablets (see Fig. 9) using Partial Least Squares (PLS) 

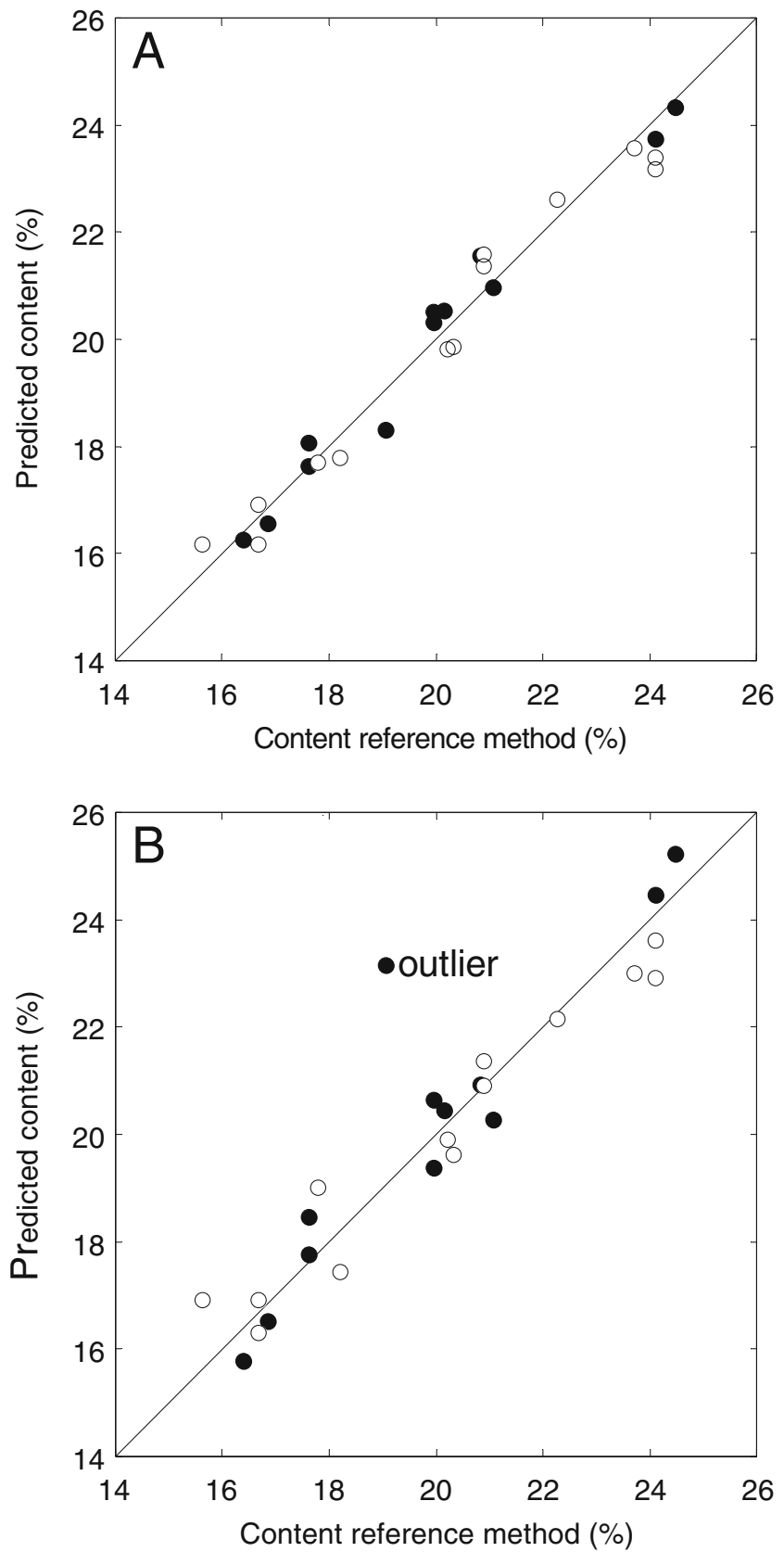

Fig. 9. Prediction of the concentration of propranolol tablets in two independent test sets; the original test sets (solid circles) and the exchanged test sets (open circles). Comparison of A Raman transmission (1 PLS component) and B Raman backscatter (1 PLS component). (Reprinted with permission from J. Johansson, A. Sparen, O. Svensson, S. Folestad, M. Claybourn, Appl. Spectrosc. 61, 1211-1218 (2007). Copyright (2007) The Society for Applied Spectroscopy (49)).

regression. The acquisition time was $10 \mathrm{~s}$ with a laser power of $400 \mathrm{~mW}(785 \mathrm{~nm})$. The measurements were also performed in a conventional backscattering Raman geometry which yielded a lower relative accuracy of $+/-2.9 \%$ under the same conditions. This was ascribed to the presence of strong sub-sampling in conventional backscattering Raman spectros-

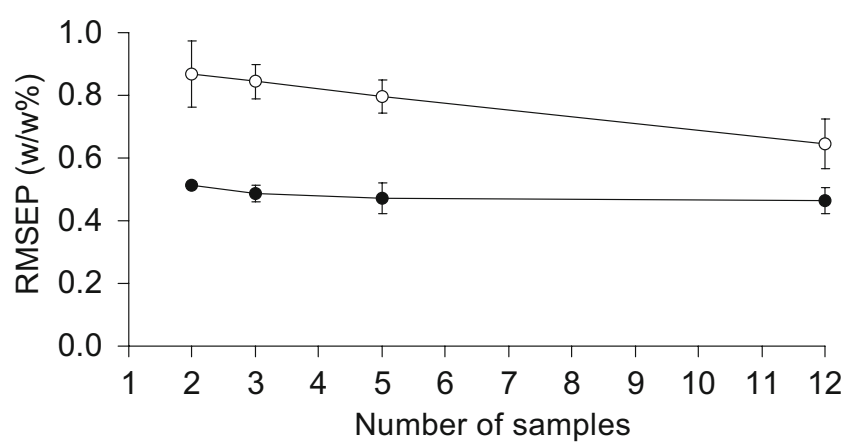

Fig. 10. Model robustness: the effect of reducing the number of samples in calibration models on the prediction errors for independent test sets. Solid circles represent the transmission mode while open circles represent the backscatter mode. The figure shows mean values of RMSEP for reduced models built on the original and the exchanged models, and the error bars show max and min values of RMSEP. The same test set was used for all reduced models originating from the same full model. (Reprinted with permission from J. Johansson, A. Sparen, O. Svensson, S. Folestad, M. Claybourn, Appl. Spectrosc. 61, 1211-1218 (2007). Copyright (2007) The Society for Applied Spectroscopy (49)).

copy and its absence with the transmission geometry. This was demonstrated on the analysis of an outlier spectrum present in the conventional backscattering Raman measurements that showed a clear deviation from the expected profile expected from the nominal concentration. No such

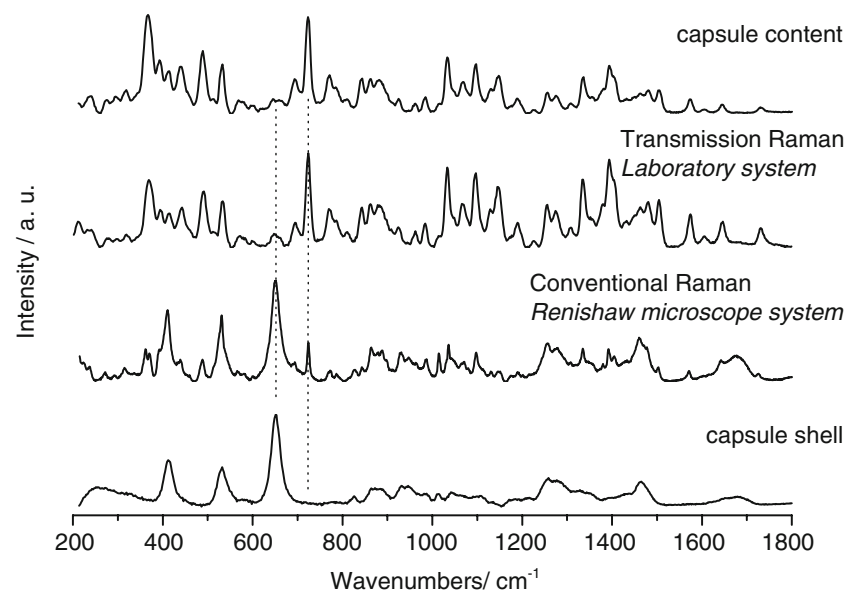

Fig. 11. Non-invasive Raman spectra of capsules. The spectra were obtained using a laboratory instrument configured in the transmission Raman geometry and a standard commercial Raman microscope (Renishaw) in conventional backscattering geometry. The Raman spectra of an empty capsule shell (lowest trace) and the capsule content itself (top trace, the capsule content was transferred into an optical cell) are shown for comparison. The dashed lines indicate the principal Raman bands of the capsule and of the API. (This figure was published in J. Pharm. Biomed. Anal., in press, C. Eliasson, N.A. Macleod, L. Jayes, F.C. Clarke, S. Hammond, M.R. Smith, P. Matousek, Non-Invasive Quantitative Assessment of the Content of Pharmaceutical Capsules using Transmission Raman Spectroscopy, Copyright Elsevier (2008) (50)). 


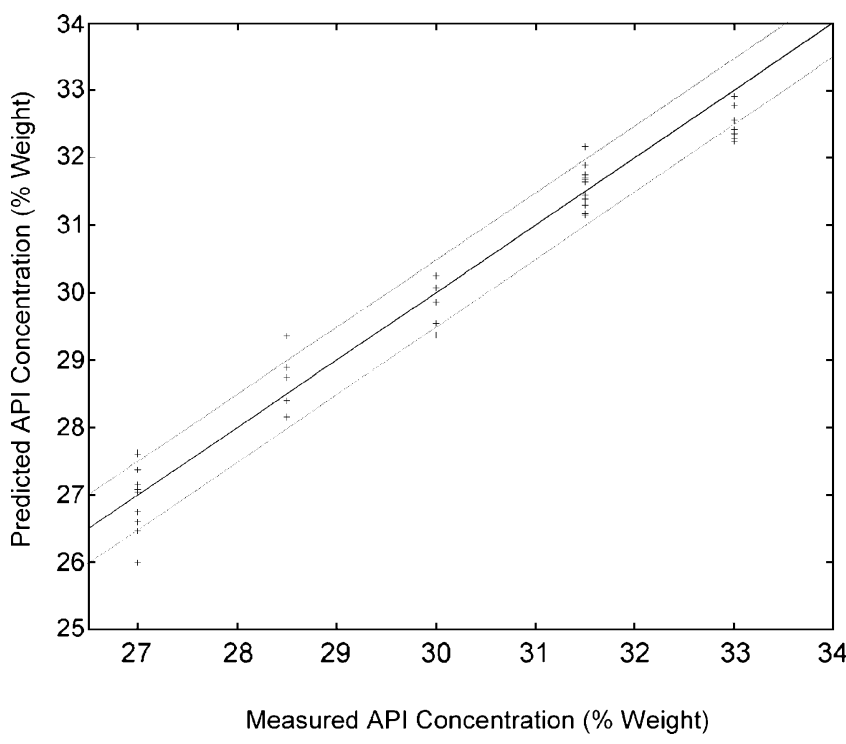

Fig. 12. Partial least squares (PLS) calibration of the API dataset $(5 \mathrm{~s}$ accumulation time). Spectra were baseline corrected, normalised to unit length and mean-centered. The dataset was randomly split into a calibration set (two thirds) and a prediction set (one third); obvious outliers from the PCA analysis were excluded from the analysis. Predicted versus measured API concentration of the prediction set, the solid line represents the $45^{\circ}$ diagonal while the dotted lines show the limits of the root mean square error of prediction. (This figure was published in J. Pharm. Biomed. Anal., in press, C. Eliasson, N.A. Macleod, L. Jayes, F.C. Clarke, S. Hammond, M.R. Smith, P. Matousek, Non-Invasive Quantitative Assessment of the Content of Pharmaceutical Capsules using Transmission Raman Spectroscopy, Copyright Elsevier (2008) (50)).

unexpected outlier was observed in the transmission geometry. It should be noted that the higher accuracy in the transmission mode was achieved despite the lower (by a factor of 10) overall Raman signal intensity. The method was also applied to pharmaceutical capsules yielding a relative accuracy of $+/-3.6 \%$.

The study also indicates that the transmission Raman mode requires a leaner calibration model (only one latent variable was required) compared with conventional backscattering Raman. The model was robust enough to provide relatively accurate predictions when only two or three spectra were used as calibration data (see Fig. 10).

In parallel research, the feasibility of quantifying the content of production line type samples prepared in a laboratory environment was demonstrated by Eliasson et al. (50). The study used 150 capsules and established the feasibility of quantifying the active pharmaceutical ingredient to within a relative error of $1-2 \%$ in transmission Raman mode. The measurements were performed through capsules containing a high level of $\mathrm{TiO}_{2}$ which prevents accurate analysis by other techniques (including conventional Raman and NIR absorption). The adoption of the transmission concept led to the suppression of this interfering signal by a factor of 33 (see Fig. 11) permitting the quantification of the API with a relative root mean square error of $+/-1.2 \%$ with $5 \mathrm{~s}$ acquisition time (see Fig. 12) per capsule. The reduction of the acquisition time to $1 \mathrm{~s}$ yielded still acceptable accuracy with the relative root mean square error increasing to $+/-1.8 \%$.

The inherent properties of the transmission geometry approach (bulk probing, gross insensitivity to alignment, suppression of interfering surface layers and non-invasive/ non-destructive probing) combined with the ability to rapidly acquire high quality spectra (within a fraction of a second) make the technique potentially well suited for implementation on production lines where both partial or complete inspection of samples is required. The handful of published studies has provided a glimpse of the potential of the technique; further research is required to provide a detailed comparison with other, more established methods (e.g. NIR) in terms of signal to noise, linearity, specificity and selectivity.

\section{CONCLUSIONS}

The emerging non-invasive, deep Raman spectroscopic techniques described in this article promise to impact on a variety of pharmaceutical analytical areas including quality and process control and the authentication of pharmaceutical products. Spatially Offset Raman Spectroscopy allows the chemical signature of pharmaceuticals to be obtained non-invasively through packaging materials for detection of counterfeit drugs and determination of shelf-life. Transmission Raman provides information on the bulk content of pharmaceutical tablets and capsules with effective suppression of interfering surface Raman and fluorescence signals allowing the rapid construction of calibration models for quantitative analysis. Signal enhancements of up to an order of magnitude can be obtained by the use of dielectric mirrors.

Perhaps most importantly, these techniques are experimentally straightforward and are readily implementable on commercial spectrometers. Although only briefly touched upon in this review, the plethora of multivariate techniques (12) applied to common spectroscopic methods (NIR, MIR etc.) are equally applicable to the results of SORS and transmission Raman providing a powerful tool for both quantitative and qualitative analysis.

Probing of deep sub-layers with Raman spectroscopy is very much a recent development and further validation studies are required to quantify, and perhaps extend the technical limitations and better define its potential. Many new and exciting applications are already visible on the horizon promising yet deeper penetration of optical analytical techniques into pharmaceutical production and post-production environments.

\section{ACKNOWLEDGEMENTS}

The authors wish to thank Dr. Darren Andrews and Dr. Tim Bestwick of CLIK Knowledge Transfer and Professor Anthony Parker and Professor Mike Dunne of the Science and Technology Facilities Council for their support of this work. The EPSRC is also gratefully acknowledged for their financial support (grant EP/D037662/1). 


\section{REFERENCES}

1. M. J. Pelletier. Analytical Applications of Raman Spectroscopy, Blackwell Science, Oxford, 1999.

2. P. Matousek, and A.W. Parker. Bulk Raman analysis of pharmaceutical tablets. Appl. Spectrosc. 60:1353-1357 (2006).

3. P. Matousek. Deep non-invasive Raman spectroscopy of living tissue and powders. Chem. Soc. Rev. 36:1292-1304 (2007).

4. B. B. Das, F. Liu, and R. R. Alfano. Time-resolved fluorescence and photon migration studies in biomedical and model random media. Rep. Prog. Phys. 60:227-292 (1997).

5. J. C. Hebden, A. Gibson, R. Yusof, N. Everdell, E. M. C. Hillman, D. T. Delpy, S. R. Arridge, T. Austin, J. H. Meek, and J. S. Wyatt. Three-dimensional optical tomography of the premature infant brain. Phys. Med. Biol. 47:4155-4166 (2002).

6. H. Koizumi, Y. Yamashita, A. Maki, T. Yamamoto, Y. Ito, H. Itagaki, and R. Kennan. Higher-order brain function analysis by trans-cranial dynamic near-infrared spectroscopy. J. Biomed. Opt. 4:403-413 (1999).

7. P. K. Owens, and J. Johansson. Light-scattering studies of packed stationary phases for capillary electrochromatography. Anal. Chem. 72:740-746 (2000).

8. T. J. Pfefer, K. T. Schomacker, M. N. Ediger, and N. S. Nishioka. Multiple-fiber probe design for fluorescence spectroscopy in tissue. Appl. Opt. 41:4712-4721 (2002).

9. N. Ghosh, S. K. Majumder, H. S. Patel, and P. K. Gupta. Depthresolved fluorescence measurement in a layered turbid medium by polarised fluorescence spectroscopy. Opt. Lett. 30:162-164 (2005).

10. L. Quan, and N. Ramanujam. Relationship between depth of target in a turbid medium and fluorescence measured by a variable-aperture method. Opt. Lett. 27:104-106 (2002).

11. S. C. Gebhart, S. K. Majumder, and A. Mahadevan-Jansen. Comparison of spectral variation from spectroscopy to spectral imaging. Appl. Opt. 46:1343-1360 (2007).

12. Paul Gemperline (ed). Practical Guide to Chemometrics, CRC, Taylor \& Francis Group, Boca Raton, Florida, 2006.

13. J. Wu, Y. Wang, L. Perelman, I. Itzkan, R. Dasari, and M. S. Feld. Three-dimensional imaging of objects embedded in turbid media with fluorescence and Raman spectroscopy. Appl. Opt. 34:3425 (1995).

14. P. Matousek, N. Everall, M. Towrie, and A. W. Parker. Depth profiling in diffusely scattering media using Raman spectroscopy and picosecond Kerr gating. Appl. Spectrosc. 59:200-205 (2005).

15. N. Everall, T. Hahn, P. Matousek, A. W. Parker, and M. Towrie. Picosecond time-resolved Raman spectroscopy of solids: capabilities and limitations for fluorescence rejection and the influence of diffuse reflectance. Appl. Spectrosc. 55:1701-1708 (2001).

16. N. Everall, T. Hahn, P. Matousek, A. W. Parker, and M. Towrie. Photon migration in Raman spectroscopy. Appl. Spectrosc. 58:591-597 (2004).

17. M. D. Morris, P. Matousek, M. Towrie, A. W. Parker, A. E. Goodship, and E. R. C. Draper. Kerr-gated time-resolved Raman spectroscopy of equine cortical bone tissue. J. Biomed. Opt. 10:014014 (2005).

18. P. Matousek, M. Towrie, A. Stanley, and A. W. Parker. Efficient rejection of fluorescence from Raman spectra using picosecond Kerr gating. Appl. Spectrosc. 53:1485-1489 (1999).

19. P. Matousek, M. Towrie, C. Ma, W. M. Kwok, D. Phillips, W. T. Toner, and A. W. Parker. Fluorescence suppression in resonance Raman spectroscopy using a high-performance picosecond Kerr gate. J. Raman Spectrosc. 32:983-988 (2001).

20. P. Matousek, M. Towrie, and A. W. Parker. Fluorescence background suppression in Raman spectroscopy using combined Kerr gated and shifted excitation Raman difference techniques. J. Raman Spectrosc. 33:238-242 (2002).

21. P. Matousek, I. P. Clark, E. R. C. Draper, M. D. Morris, A. E Goodship, N. Everall, M. Towrie, W. F. Finney, and A. W. Parker. Subsurface probing in diffusely scattering media using spatially offset Raman spectroscopy. Appl. Spectrosc. 59:393-400 (2005).

22. P. Matousek, M. D. Morris, N. Everall, I. P. Clark, M. Towrie, E. Draper, A. Goodship, and A. W. Parker. Numerical simulations of subsurface probing in diffusely scattering media using spatially offset Raman spectroscopy. Appl. Spectrosc. 59:1485-1492 (2005).

23. W. Chew, E. Widjaja, and M. Garland. Band-target entropy minimization (BTEM): an advanced method for recovering unknown pure component spectra. Application to the FTIR spectra of unstable organometallic mixtures. Organometallics. 21:1982-1990 (2002).

24. E. Widjaja, N. J. Crane, T. C. Chen, M. D. Morris, M. A. Ignelzi, and B. R. McCreadie. Band-target entropy minimization (BTEM) applied to hyperspectral Raman image data. Appl. Spectrosc. 57:1353-1362 (2003)

25. M. V. Schulmerich, K. A. Dooley, T. M. Vanasse, S. A. Goldstein, and M. D. Morris. Subsurface and transcutaneous Raman spectroscopy and mapping using concentric illumination rings and collection with a circular fiber-optic array. Appl. Spectrosc. 61:671-678 (2007).

26. C. Eliasson, N. A. Macleod, and P. Matousek. Noninvasive detection of concealed liquid explosives using Raman spectroscopy. Anal. Chem. 79:8185-8189 (2007).

27. C. Eliasson, and P. Matousek. Noninvasive authentication of pharmaceutical products through packaging using spatially offset Raman spectroscopy. Anal. Chem. 79:1696-1701 (2007).

28. J. Y Ma, and D. Ben-Amotz. Rapid micro-Raman imaging using fiber-bundle image compression. Appl. Spectrosc. 51:1845-1848 (1997).

29. M. V. Schulmerich, W. F. Finney, V. Popescu, M. D. Morris, T. M. Vanasse, and S. A. Goldstein. Transcutaneous Raman spectroscopy of bone tissue using a non-confocal fiber optic array probe. In A. Mahadevan-Jansen and W. H. Petrich (eds), Proceedings of SPIE 6093, Biomedical Vibrational Spectroscopy III: Advances in Research and Industry, 2006, 60930O.

30. M. V. Schulmerich, W. F. Finney, R. A. Fredricks, and M. D. Morris. Subsurface Raman spectroscopy and mapping using a globally illuminated non-confocal fiber-optic array probe in the presence of Raman photon migration. Appl. Spectrosc. 60:109114 (2006).

31. P. Matousek, E. R. C. Draper, A. E. Goodship, I. P. Clark, K. L. Ronayne, and A. W. Parker. Noninvasive Raman spectroscopy of human tissue in vivo. Appl. Spectrosc. 60:758-763 (2006).

32. C. Eliasson, M. Claybourn, and P. Matousek. Deep subsurface Raman spectroscopy of turbid media by a defocused collection system. Appl. Spectrosc. 61:1123-1127 (2007).

33. P. Matousek. Inverse spatially offset Raman spectroscopy for deep noninvasive probing of turbid media. Appl. Spectrosc. 60:1341-1347 (2006).

34. M. V. Schulmerich, K. A. Dooley, M. D. Morris, T. M. Vanasse, and S. A. Goldstein. Transcutaneous fiber optic Raman spectroscopy of bone using annular illumination and circular array of collection fibers. J. Biomed. Opt. 11:060502 (2006).

35. M. V. Schulmerich, M. D. Morris, T. M. Vanasse, and S. A. Goldstein, Transcutaneous Raman spectroscopy of bone global sampling and ring/disk fiber optic probes. In T. Vo-Dinh, W.S. Grundfest, D.A. Benaron, G.E. Cohn and R. Raghavachari (eds), Proceedings of SPIE 6430, Advanced Biomedical and Clinical Diagnostic Systems V, 2007, 643009.

36. B. Depret, P. Verkerk, and D. Hennequin. Characterization and modelling of the hollow beam produced by a real conical lens. Opt. Commun. 211:31-38 (2002).

37. B. Schrader, and G. Bergmann. Die intensitat des Ramanspektrums polykristalliner substanzen. I. Strahlungsbilanz von substanz und probenanordnung. Zeitschrift fur Analytische Chemie Fresenius. 225:230-247 (1967).

38. J. Johansson, S. Pettersson, and S. Folestad. Characterization of different laser irradiation methods for quantitative Raman tablet assessment. J. Pharm. Biomed. Anal. 39:510-516 (2005).

39. P. Matousek, and A. W. Parker. Non-invasive probing of pharmaceutical capsules using transmission Raman spectroscopy. J. Raman Spectrosc. 38:563-567 (2007).

40. P. Matousek. Raman signal enhancement in deep spectroscopy of turbid media. Appl. Spectrosc. 61:845-854 (2007).

41. C. Eliasson, and P. Matousek. Passive signal enhancement in spatially offset Raman spectroscopy. J. Raman Spectrosc. (2008) (in press). DOI 10.1002/jrs.1898 
42. R. Cockburn, P. N. Newton, E. K. Agyarko, D. Akunyili, and N. J. White. The global threat of counterfeit drugs: why industry and governments must communicate the dangers. PLoS Medicine. 4:1553-1553 (2007).

43. P. Aldhous. Counterfeit pharmaceuticals: murder by medicine. Nature. 434:132-136 (2005).

44. Counterfeit medicines. Guidance for pharmacists. Royal Pharmaceutical Society of Great Britain. http://www.rpsgb.org.uk/ pdfs/counterfeitmedsguid.pdf. (accessed 1/8/2008).

45. P. N. Newton, M. D. Green, F. M. Fernández, N. P. J. Day, and N. J. White. Counterfeit anti-infective drugs. Lancet Infect. Dis. 6:602-613 (2006).

46. M. R. Witkowski. The use of Raman spectroscopy in the detection of counterfeit and adulterated pharmaceutical products. Am. Pharm. Rev. 8:56-60 (2005).
47. C. Eliasson, and P. Matousek. Spatial offset broadens applications for Raman spectroscopy. Laser Focus World. 43:123 (2007).

48. C. Ricci, C. Eliasson, N. A. Macleod, P. Newton, P. Matousek, and S. G. Kazarian. Characterization of genuine and fake artesunate anti-malarial tablets using Fourier transform infrared imaging and spatially offset Raman spectroscopy through blister packs. Anal. Bioanal. Chem. 389:1525-1532 (2007).

49. J. Johansson, A. Sparen, O. Svensson, S. Folestad, and M. Claybourn. Quantitative transmission Raman spectroscopy of pharmaceutical tablets and capsules. Appl. Spectrosc. 61:1211-1218 (2007).

50. C. Eliasson, N. A. Macleod, L. Jayes, F. C. Clarke, S. Hammond, M. R. Smith, P. Matousek, Non-Invasive quantitative assessment of the content of pharmaceutical capsules using transmission Raman spectroscopy. J. Pharm. Biomed. Anal. (2008) (in press). DOI 10.1016/j.jpba.2008.01.013 